\title{
Lattice Distortion Mediated Paramagnetic Relaxation in High-Spin High-Symmetry Molecular Magnets
}

\author{
Anupam Garg \\ Department of Physics and Astronomy, Northwestern University, Evanston, Illinois 60208
}

(May 11, 1998)

\begin{abstract}
Field-dependent maxima in the relaxation rate of the magnetic molecules $\mathrm{Mn}_{12}$-Ac and $\mathrm{Fe}_{8}$-tacn have commonly been ascribed to some resonant tunneling phenomenon. We argue instead that the relaxation can be understood as purely due to phonons. The rate maxima arise because of a Jahn-Tellerlike distortion caused by the coupling of lattice phonons to degenerate Zeeman levels of the molecule at the top of the barrier. The binding energy of the distorted intermediate states lowers the barrier height and increases the relaxation rate. A nonperturbative calculation of this effect is carried out for a model system. An approximate result for the field variation near a maximum is found to agree reasonably with experiment.
\end{abstract}

76.30.-v, 76.20.+q, 75.80.+q, 75.60.Ej 
In the last five years, a remarkable and novel type of paramagnetic relaxation has been observed in certain molecular crystals 14 . The best known, a $\mathrm{Mn}_{12}$-acetate complex (or just $\mathrm{Mn}_{12}$ for short), has a ground state spin $S=10$, and a four-fold symmetry axis $\mathbf{z}$, which coincides with the $\mathbf{c}$ axis of the tetragonal crystal that it forms. It is found that if the spin is first polarized along $\mathbf{c}$, and then allowed to relax by reversing the field to a value $H$, the relaxation rate is a non monotonic function of $H$, showing maxima at roughly equally spaced fields $H_{n}=n H^{*}$, with $H^{*}=0.44 \mathrm{~T}$. Hysteresis loops measured with a slow field sweep show sharp jumps in the magnetization $M$ at the same reverse fields $H_{n}$. The relaxation rate $\Gamma$ obeys an Arrhenius law with a barrier of $\sim 62 \mathrm{~K}$ when $H=0$. Similar effects have been seen in $\left[(\operatorname{tacn})_{6} \mathrm{Fe}_{8} \mathrm{O}_{2}(\mathrm{OH})_{1} 2\right]^{8}+$, though not as sharply.

In this Letter we contend that at present there is not even a qualitative understanding of these rate maxima, and attempt to fill this gap. We begin by reviewing the basic background theory, which is due to Villain, Hartmann-Boutron, Sessoli, and Rettori (VHSR) [5]. The principle terms in the spin Hamiltonian are

$$
\mathcal{H}_{s}=-D S_{z}^{2}-h S_{z}
$$

where $h=g \mu_{B} H$. The next order terms, $S_{z}^{4}$ and $S_{ \pm}^{4}$, are small and have been ignored. The spin states $|m\rangle$, where $S_{z}|m\rangle=m|m\rangle$, have energies $\epsilon_{m}=-D m^{2}-h m$. Suppose the initial spin state is $|-S\rangle$, and $\epsilon_{-S}>\epsilon_{S}$. As explained by VHSR, the spin relaxes via a multistep Orbach process. It absorbs phonons and climbs from $m=-S$ to $-S+1,-S+2, \ldots$, until it reaches the highest level $m^{*}$, and then descends to levels $m^{*}+1, m^{*}+2, \ldots, S$ by emitting phonons. The occupation probabilities $\left\{p_{m}\right\}$ obey a set of master equations

$$
\frac{d p_{m}}{d t}=-\sum_{n} \gamma_{m}^{n} p_{m}+\sum_{n} \gamma_{n}^{m} p_{n}
$$

where $\gamma_{m}^{n}$ is the rate or probability per unit time for the $m \rightarrow n$ transition. To calculate $\left\{\gamma_{m}^{n}\right\}$, VHSR use Fermi's golden rule with a standard spin-phonon interaction

$$
\mathcal{H}_{\mathrm{sp}}=\sum_{\alpha} g_{i j k l} \omega_{\alpha}^{-1 / 2} e_{\alpha i} q_{\alpha j}\left(a_{\alpha}+a_{\alpha}^{\dagger}\right)\left(S_{k} S_{l}+S_{l} S_{k}\right)
$$

where $\alpha$ labels the phonon modes with creation and anihilation operators $a_{\alpha}^{\dagger}$ and $a_{\alpha}, \mathbf{q}_{\alpha}, \mathbf{e}_{\alpha}$, and $\omega_{\alpha}$ are the wavevector, polarization, and frequency, and $i, j$, etc. are Cartesian indices. The tensor $g_{i j k l}$ is only weakly q dependent. With $\epsilon_{m n} \equiv \epsilon_{m}-\epsilon_{n}$, we obtain

$$
\gamma_{m}^{n}=K\left|V_{m n}\right|^{2} \times \begin{cases}\epsilon_{n m}^{3} n\left(\epsilon_{n m}\right), & \epsilon_{m}<\epsilon_{n}, \\ \epsilon_{m n}^{3}\left[1+n\left(\epsilon_{m n}\right)\right], & \epsilon_{m}>\epsilon_{n} .\end{cases}
$$

Here, $K$ is a constant dependent on the sound speed, the density of the solid, and the couplings $g_{i j k l}, V_{m n}$ denotes a spin matrix element such as $\left\langle m\left|S_{k} S_{l}\right| n\right\rangle$, and $n(\epsilon)$ is the Bose function $n(\epsilon)=\left(e^{\beta \epsilon}-1\right)^{-1}$, with $\beta=1 / k_{B} T$. VHSR solve these rate equations and find that for $H=0$,

$$
\Gamma=K\left|V_{10}\right|^{2} D^{3} \exp \left(-\beta D S^{2}\right) /\left(1-e^{-\beta D}\right) .
$$

The factor $e^{-\beta D S^{2}}$ yields the expected activated behavior with an energy barrier $D S^{2}$. The spin phonon interaction required to fit the observed rate is somewhat large, but not unreasonable. 
Since the relaxation is a sequential process in this picture, $\Gamma$ is limited by the weakest link in the chain [7]. This leads to a disaster whenever the field is such that the topmost two levels, $m$ and $m^{\prime}$, say, are degenerate. For then, by Eq. (本), $\gamma_{m^{\prime}}^{m}$ and $\gamma_{m}^{m^{\prime}}$ vanish as $k_{B} T \epsilon_{m m^{\prime}}^{2}$. One link in the chain is broken, and so $\Gamma$ must vanish completely!

In fact this conclusion is not correct in detail. To see this, we first note that the $S_{x} S_{z}$ and $S_{y} S_{z}$ terms in Eq. (3) connect levels with $\Delta m=1$, while $S_{x} S_{y}$ like terms allow $\Delta m=2$ processes. (The $\Delta m=0$ terms lead to ignorable level shifts.) Second, there are two types of level crossings: $m$ and $m+1$ coincide $\left(\Delta m=1\right.$ or "odd" crossings) when $h=h_{-(2 m+1)}$, and $m$ and $m+2$ coincide $\left(\Delta m=2\right.$ or "even" crossings) when $h=h_{-(2 m+2)}$, where $h_{j} \equiv j D$. Since the $\Delta m=2$ channel is open at a $\Delta m=1$ crossing, and vice versa, relaxation is still possible. Nevertheless, $\Gamma$ should be a minimum whenever $h=h_{n}$ for any $n$, even or odd, as one channel is closed off. This still poses a problem, as these are just the field values where the observed $\Gamma$ is a maximum. Taking $D=0.57 \mathrm{~K}$ from electron spin resonance (ESR) experiments [6], we get $H_{j}=0.42 j \mathrm{~T}$, which agrees quite well with the measured jump fields in the hysteresis loops.

Given the above agreement, it is extremely tempting to argue that there is a resonant coupling between degenerate energy levels near the top of the barrier [8] due to some perturbation, such as hyperfine or dipole-dipole interaction, or terms such as $S_{+}^{4}+S_{-}^{4}$ in the spin Hamiltonian [9, 10]. By and large, all these mechanisms are either too weak to explain the observed widths of $0.03-0.05 \mathrm{~T}$ in the relaxation rate maxima, and/or run into difficulties with selection rules. The hyperfine field, e.g., has an estimated rms strength of $0.015 \mathrm{~T}$, and must act in second order to connect levels with $\Delta m=2$, which implies a width $\sim 10^{-3}$ T. Similarly, the dipolar field is estimated as $\sim 0.01 \mathrm{~T}$. Not only is this again a little small, but an experiment on a frozen solution of $\mathrm{Mn}_{12}$ [1] with greater intermolecular separation than the crystal shows faster relaxation, exactly the opposite of what would be expected. The $S_{ \pm}^{4}$ terms cannot explain the $\Delta m=1$ maxima. The strongest evidence that a perturbation involving only the spin is not responsible for the rate maxima comes from experiments in which the field is applied at an angle $\theta$ to the $z$ axis 12,13. Since the perturbation $-h_{x} S_{x} \propto \theta$ for small $\theta, \Gamma$ should rise dramatically as $\theta$ increasess from say $0.5^{\circ}$ to $10^{\circ}$ [14, with $H_{z}$ held fixed at $H_{n}$. However, as can be seen from Fig. 4 of Ref. [13], the jump $\Delta M$ hardly changes at all for $H_{z}=H_{1}$, and increases only by 2 at $H_{z}=H_{2}$ as $\theta$ is varied from 0 to $10^{\circ}$. Further, the width of the jump at $H_{1}$ is essentially unchanged even as $\theta$ is increased to $44^{\circ}$, whereas a simple anticrossing would yield a width varying as $D \sin \theta$.

How, then, does the magnetization relax when $H=H_{n}$ ? In our view, the causative agency is still the spin-phonon interaction but it now plays a dual role. Specifically, the part of $\mathcal{H}_{\mathrm{sp}}$ that couples two levels can not be treated perturbatively when these two levels are degenerate. One way to see this is to map the degenerate levels onto the $s_{z}= \pm 1 / 2$ levels of a pseudospin with $s=1 / 2$, to write $\mathcal{H}_{\text {sp }}$ and $\mathcal{H}_{p}$ as $\sum c_{\alpha} s_{x} x_{\alpha}$ and $\sum \omega_{\alpha}\left(p_{\alpha}^{2}+x_{\alpha}^{2}\right) / 2$, where $x_{\alpha}$ and $p_{\alpha}$ are oscillator position and momentum coordinates for mode $\alpha$. It is then obvious that the lowest energy eigenstates are those of $s_{x}$, not $s_{z}$, and that in these states the oscillators have a non zero mean displacement, $\left\langle x_{\alpha}\right\rangle=-c_{\alpha} s_{x} / \omega_{\alpha}$. The binding energy $\Omega$ due to this displacement, effectively leads to a reduction of the net barrier, and hence to an enhanced relaxation rate. 


\section{FIGURES}

FIG. 1.

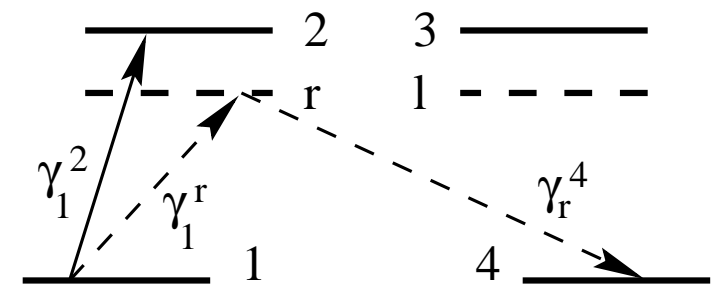

Upper four magnetic levels in the degenerate case, without (solid lines) and with (dashed lines) lattice distortion.

In the rest of the Letter we shall sketch a calculation of this effect for a model problem, in which the spin-phonon interaction is not the most general one possible, but which would also yield a vanishing rate at degeneracy if treated perturbatively. We will show that phonons alone give a nonzero rate, which in fact exceeds that which would be guessed from a Arrhenius law which the barrier varies smoothly with $H$ and the prefactor is taken as constant. We will also show that the rate is a local maximum at degeneracy, with an approximate field dependence that describes the data on $\mathrm{Mn}_{12}$ fairly well.

Our model focuses on the four energy levels near the top of the barrier (see Fig. 1), which we label $1, \ldots, 4$. The full Hamiltonian $\mathcal{H}$ is the sum of spin, spin-phonon, and phonon terms:

$$
\begin{aligned}
\mathcal{H}_{s} & =\sum_{j=1}^{4} \epsilon_{j}|j\rangle\langle j| \\
\mathcal{H}_{\mathrm{sp}} & =\sum_{\alpha, j} a_{j, j+1}\left(t_{j, j+1}+t_{j+1, j}\right) c_{\alpha} x_{\alpha} \\
\mathcal{H}_{p} & =\sum_{\alpha} \frac{\omega_{\alpha}}{2}\left(p_{\alpha}^{2}+x_{\alpha}^{2}\right)
\end{aligned}
$$

where $t_{i j}=|i\rangle\langle j|$, and we have set $\hbar=1$. We will take $\epsilon_{1} \approx \epsilon_{4}, \epsilon_{2} \approx \epsilon_{3}$, and make the inessential simplification $a_{34}=a_{12}$. Also, we choose a spectral density

$$
J(\omega) \equiv \frac{\pi}{2} \sum_{\alpha} c_{\alpha}^{2} \delta\left(\omega-\omega_{\alpha}\right)=B \omega^{3} \exp \left(-\omega / \omega_{c}\right)
$$

which describes a coupling to accoustic phonons as per by Eq. (3). The cutoff $\omega_{c}$ is like a Debye frequency. Defining $D=\epsilon_{21}$, we assume $\omega_{c} \gg D, k_{B} T$, and also that the maximal lattice distortion binding energy $\Omega$ (realized in the case of exact degeneracy $\epsilon_{2}=\epsilon_{3}$ ) obeys $\Omega \ll D$. This energy is given by

$$
\Omega=\frac{a_{23}^{2}}{\pi} \int_{0}^{\infty} d \omega \frac{J(\omega)}{\omega}=a_{23}^{2} \sum_{\alpha} \frac{c_{\alpha}^{2}}{2 \omega_{\alpha}} .
$$

Note that since all oscillators contribute to $\Omega$ and not just those near $\omega=0$, the high frequency details of $J(\omega)$ can significantly affect $\Omega$.

We now wish to calculate $\gamma_{1}^{4}$, the net rate at which the spin, initially in state $|1\rangle$, relaxes to state $|4\rangle$, at a temperature $T$. For future use and for comparison with Eq. (止), we record here the result of a Fermi golden rule calculation of the $1 \rightarrow 2$ transition rate: 


$$
\gamma_{\mathrm{abs}}^{0}=2 a_{12}^{2} J(D) n(D) \approx 2 B a_{12}^{2} D^{3} /\left(e^{\beta D}-1\right) .
$$

A similar golden rule calculation gives $\gamma_{2}^{3}=0$, and as discussed earlier, utterly fails to explain how the spin relaxes. The correct procedure is to first find the exact eigenstates of the full Hamiltonian in the 23 subspace, $\mathcal{H}_{23}=P_{23} \mathcal{H} P_{23}, P_{23}=|2\rangle\langle 2|+| 3\rangle\langle 3|$, and then find the absorption and emission rates to and from these intermediate states by the golden rule. The absorption rate from state 1 so calculated can be formally written as

$$
\gamma_{\mathrm{abs}}=2 \pi \sum_{\mu} p_{\mu}\left\langle 1, \mu\left|V^{\dagger} \delta\left(\epsilon_{1}+\epsilon_{\mu}-\mathcal{H}_{23}\right) V\right| 1, \mu\right\rangle,
$$

where $V=a_{12} \sum_{\alpha} c_{\alpha} x_{\alpha}|2\rangle\langle 1|$, and $\mu$ labels the initial phonon states, with energy $\epsilon_{\mu}$, and occurrence probability $p_{\mu} \propto \exp \left(-\beta \epsilon_{\mu}\right)$. The emission rate can be similarly expressed.

Formula (12) can be greatly simplified when $\epsilon_{2}=\epsilon_{3}$. Fourier transforming the energy $\delta$-function, and expressing the sum over $\mu$ in terms of the density matrix $\exp \left(-\beta \mathcal{H}_{p}\right)$, we obtain a trace over phonons which involves only Gaussian integrals. A lengthy but simple analysis yields

$$
\gamma_{\mathrm{abs}}=a_{12}^{2} \int_{-\infty}^{\infty} d \tau e^{-i\left[D \tau-a_{23}^{2} R(\tau)\right]}\left[S_{1}(\tau)+a_{23}^{2} S_{2}^{2}(\tau)\right]
$$

where

$$
\begin{aligned}
S_{1}(\tau) & =\frac{1}{\pi} \int_{0}^{\infty} d \omega J(\omega)\left(f_{a}+f_{e}\right), \\
S_{2}(\tau) & =\frac{1}{\pi} \int_{0}^{\infty} d \omega \frac{J(\omega)}{\omega}\left(1+f_{a}-f_{e}\right), \\
R(\tau) & =\frac{1}{\pi} \int_{0}^{\infty} d \omega \frac{J(\omega)}{\omega^{2}}\left[\omega \tau+i \operatorname{coth} \frac{\beta \omega}{2}-i\left(f_{a}+f_{e}\right)\right],
\end{aligned}
$$

with $f_{a}=e^{i \omega \tau} n(\omega)$, and $f_{e}=e^{-i \omega \tau}[1+n(\omega)]$.

The essential aspects of the integral (13) follow from the behavior of $a_{23}^{2} R(\tau)$ as $\tau \rightarrow \infty$. The first term in Eq. (16) grows linearly with $\tau$, the second tends to a constant, and the last two vanish as $\tau \rightarrow \infty$. We make this explicit by writing

$$
R(\tau)=a_{23}^{-2}(\Omega \tau+i W)-i R^{\prime}(\tau)
$$

where $\Omega$ is the binding energy (10),

$$
W=\frac{a_{23}^{2}}{\pi} \int_{0}^{\infty} d \omega \frac{J(\omega)}{\omega^{2}} \operatorname{coth} \frac{\beta \omega}{2},
$$

and $R^{\prime}(\tau)=\int d \omega J(\omega)\left(f_{a}+f_{e}\right) / \pi \omega^{2}$. The term $\Omega \tau$ clearly must be combined with $D \tau$ in the exponential factor in Eq. (13). This point makes it obvious that the relevant transition energy when $\epsilon_{32}=0$ is not the bare $D$, but rather the reduced quantity

$$
\widetilde{D}=D-\Omega \text {. }
$$

Likewise, the term $W$ reduces the coupling constant $a_{12}$ to $\tilde{a}_{12}=a_{12} \exp (-W / 2)$. This is nothing but the Franck-Condon overlap factor between the unpolarized and polarized lattice states accompanying the spin states 1 and 2 (or 3), respectively. 
The explicit form (9) for $J(\omega)$ yields $\Omega=2 B a_{23}^{2} \omega_{c}^{3} / \pi$, and $W \approx B a_{23}^{2} \omega_{c}^{2} / \pi=2 \Omega / \omega_{c}$ for $k_{B} T \ll \omega_{c}$. Since $\Omega<D$ and $D \ll \omega_{c}$ by assumption, $W \ll 1$.

We can further approximate Eq. (13) by expanding the remaining factor $\exp \left[a_{23}^{2} R^{\prime}(\tau)\right]$ in powers of $a_{23}$ up to $O\left(a_{23}^{2}\right)$, which is justified as long as $a_{23}$ is not too large, and reflects what we mean by the "weak" non-perturbative limit. If this is done, $\gamma_{\mathrm{abs}}$ can be written as the sum of "direct" and "indirect" parts [15],

$$
\begin{aligned}
\gamma_{\text {dir }} & =\tilde{a}_{12}^{2} \int_{-\infty}^{\infty} d \tau e^{-i \widetilde{D} \tau} S_{1}(\tau)=2 \tilde{a}_{12}^{2} J(\widetilde{D}) n(\widetilde{D}), \\
\gamma_{\text {in }} & =\tilde{a}_{12}^{2} a_{23}^{2} \int_{-\infty}^{\infty} d \tau e^{-i \widetilde{D} \tau}\left[S_{2}^{2}(\tau)+S_{1}(\tau) R^{\prime}(\tau)\right] .
\end{aligned}
$$

The last result for $\gamma_{\text {dir }}$ follows by first doing the $\tau$ integral, and noting that $\widetilde{D}>0$, so only the absorption term $f_{a}$ in Eq. (14) contributes. A similar procedure shows that $\gamma_{\text {in }}$ can be expressed as the sum of three parts,

$$
\begin{aligned}
& \gamma_{\mathrm{in}, 1}=4 \tilde{a}_{12}^{2} \frac{\Omega}{\widetilde{D}} J(\widetilde{D}) n(\widetilde{D}), \\
& \gamma_{\mathrm{in}, 2}=\frac{1}{\pi} \tilde{a}_{12}^{2} a_{23}^{2} \widetilde{D}^{2} \iint d \omega_{1} d \omega_{2} \frac{J\left(\omega_{1}\right) J\left(\omega_{2}\right)}{\omega_{1}^{2} \omega_{2}^{2}} n\left(\omega_{1}\right) n\left(\omega_{2}\right) \delta\left(\omega_{1}+\omega_{2}-\widetilde{D}\right), \\
& \gamma_{\mathrm{in}, 3}=\frac{2}{\pi} \tilde{a}_{12}^{2} a_{23}^{2} \widetilde{D}^{2} \iint d \omega_{1} d \omega_{2} \frac{J\left(\omega_{1}\right) J\left(\omega_{2}\right)}{\omega_{1}^{2} \omega_{2}^{2}} n\left(\omega_{1}\right)\left[1+n\left(\omega_{2}\right)\right] \delta\left(\omega_{1}-\omega_{2}-\widetilde{D}\right) .
\end{aligned}
$$

It can now be shown that $\gamma_{\text {in, } 2} / \gamma_{\text {in, } 1} \sim\left(\widetilde{D} / \omega_{c}\right)^{3}$ for $k_{B} T \ll \widetilde{D}$, and $\sim \widetilde{D}^{2} k_{B} T / \omega_{c}^{3}$ for $k_{B} T \gg$ $\widetilde{D}$, while $\gamma_{\mathrm{in}, 3} / \gamma_{\mathrm{in}, 1} \sim \widetilde{D}\left(k_{B} T\right)^{2} / \omega_{c}^{3}$ for all $T$. It is assumed that $k_{B} T \ll \omega_{c}$ always. Dropping $\gamma_{\mathrm{in}, 2}$ and $\gamma_{\mathrm{in}, 3}$ completely, we obtain the compact approximation [compare with Eq. (11)],

$$
\begin{aligned}
\gamma_{\mathrm{abs}} & \approx 2 \tilde{a}_{12}^{2}(1+2 \Omega / \widetilde{D}) J(\widetilde{D}) n(\widetilde{D}) \\
& =2 B \tilde{a}_{12}^{2} \widetilde{D}^{3}(1+2 \Omega / \widetilde{D}) /\left(e^{\beta \widetilde{D}}-1\right) .
\end{aligned}
$$

Note that $\gamma_{\text {in }} \ll \gamma_{\text {dir }}$ because $\Omega \ll D$, which renders the expansion in $a_{23}$ sensible. The condition $\Omega \ll D$ is actually basic to the very formulation of our calculation, for otherwise the levels $|1\rangle$ and $|4\rangle$ will also induce significant polarization of the lattice, and treating them as bare levels is unjustified.

The last step is to find the over all rate $\gamma_{1}^{4}$. Let us do this first for the symmetric case, where $\epsilon_{32}=\epsilon_{41}=0$. Then, phonon absorption starting from state $|1\rangle$ takes the spin to states $|r, l\rangle \equiv 2^{-1 / 2}(|2\rangle \pm|3\rangle)$. The individual rates for these transitions clearly obey $\gamma_{1}^{r}=\gamma_{1}^{l}=\gamma_{\mathrm{abs}} / 2$. Liekwise the rates for phonon emission from these levels to level 4 are equal, and given by $\gamma_{r}^{4}=\gamma_{l}^{4}=e^{\beta \widetilde{D}} \gamma_{\text {abs }} / 2$ using detailed balance. Following Ref. [5], one finds

$$
\gamma_{1}^{4}=\gamma_{\mathrm{abs}}
$$

We thus see that even with degenerate top levels, phonons can lead to spin relaxation. In fact the rate (26) exceeds that given by a classical Arrhenius law in which the field dependence is smooth and enters only through the overall energy barrier. In making this comparison, let us also incorporate the lower $2 S-3$ energy levels into the picture. The net 
rate is again determined by the slowest step, and at low temperatures, $\exp (-\beta \widetilde{D}) \ll 1$, it can be found by multiplying $\gamma_{1}^{4}$ by the Boltzmann factor for level 1 . Ignoring factors of order unity, and writing the matrix element as $|V|^{2}$, we have

$$
\Gamma \simeq K|V|^{2} \widetilde{D}^{2}(\widetilde{D}+2 \Omega) e^{-[W+\beta(U-\Omega)]}
$$

where $U=\epsilon_{1}-\epsilon_{-S}$ is the full energy barrier. This should be compared with a "naive" rate, $\Gamma^{0} \simeq K|V|^{2} D^{3} e^{-\beta U}$. It is not hard to see that $\Gamma>\Gamma^{0}$ provided $k_{B} T<\min \left(\omega_{c}, D^{2} / \Omega\right)$ [16.

We can also ask for the rate in the asymmetric case, when $\epsilon_{32} \neq 0$. We do not now have closed form answers, and our results are more approximate. Let $\epsilon_{2}=-\epsilon_{3}=\zeta / 2$, and $\epsilon_{1}=-\epsilon_{4}=\zeta$, where $\zeta \ll D$. Lattice polarization will now change $\zeta$ to $\zeta e^{-W}$, and the spin states will be slightly rotated from $|r, l\rangle$. The main effect, however, will be that the binding energy itself is reduced: $\Omega \rightarrow \Omega-c \zeta^{2} / \Omega$, where $c=O(1)$. This means that the center of gravity of the levels 2 and 3 will not be lowered as much as in the degenerate case. Secondly, we can safely approximate the rates $\gamma_{1}^{r}$, etc. by the form (25) (times 1/2), but with the correct energy differences in the Bose factors. The rate $\gamma_{1}^{4}$ can now be written as a sum of rates through the parallel channels $|r\rangle$ and $|l\rangle$. A straightforward analysis shows that the terms linear in $\zeta$ (due to differences in $\gamma_{1}^{r}$ and $\gamma_{1}^{l}$, e.g.) cancel. The chief surviving term is from the systematic reduction in binding energy, leading to a factor $\exp \left(-c \beta \zeta^{2} / \Omega\right)$ in $\gamma_{1}^{4}$. This shows that the relaxation rate has a local maximum near the coincidence fields [17].

We conclude by asking how far our model applies to real $\mathrm{Mn}_{12}$. The obvious omissions are (a) $\Delta m=2$ processes, and (b) $t_{j, k}-t_{k, j}$ couplings in Eq. (7). Neither of these is expected to change the qualitative picture given here. The key unknowns are the binding energy $\Omega$, and whether $\Omega \ll D$. A look at Fig. 2 of Ref. $[13$ shows that a field variation of the form $\Gamma(H) \sim \exp \left(-c \beta \zeta^{2} / \Omega\right)$ with $\zeta=\Delta m\left(h-h_{n}\right)$, describes the rate maxima quite well, and we find $\Omega / c=9,6,10$, and $4 \mathrm{mK}$ for $n=0,1,2$, and 3, respectively. Direct estimates based, e.g., on Eq. (B.3) of Ref. [10], yield $\Omega$ values $0.5-20 \mathrm{mK}$, which are quite consistent given the uncertainties in $\omega_{c}$ etc. Direct experimental probes of $\mathcal{H}_{\mathrm{sp}}$ are clearly very important to testing our theory. Suggestions for how this may be done, and more detailed calculations will be published elsewhere.

I am indebted to B. Barbara, A. J. Leggett, C. Paulsen, M. Randeria, and R. Sessoli for many useful discussions. This work was done in part at the Institute for Nuclear Theory, University of Washington, Seattle, and I thank the memebrs and staff of the Institute for their warm hospitality. This work is supported by the National Science Foundation through grant number DMR-9616749. 


\section{REFERENCES}

* Electronic address: agarg@nwu.edu

[1] M. A. Novak and R. Sessoli, in Quantun Tunneling of Magnetization-QTM'94, edited by L. Gunther and B. Barbara (Kluwer, Dordecht, 1995).

[2] J. Friedman, M. P. Sarachik, J. Tejada, and R. Ziolo, Phys. Rev. Lett. 76, 3830 (1996).

[3] L. Thomas et al., Nature 383, 145 (1996).

[4] C. Sangregorio et al., Phys. Rev. Lett. 78, 4645 (1997).

[5] J. Villain, F. Hartman-Boutron, R. Sessoli, and A. Rettori, Europhys. Lett. 27, 159 (1994).

[6] A. L. Barre, D. Gatteschi, and R. Sessoli, Phys. Rev. B 56, 8192 (1997).

[7] Thus, Eq. (5) can be understood as the Boltzmann factor for occupation of the level $m=-1$, times the rate $\gamma_{-1}^{0}$.

[8] The simultaneous degeneracy of lower pairs of levels when $h=n D$ implied by Eq. (1) is surely artificial. ESR indicates a correction term $B\left(S_{x}^{2}-S_{y}^{2}\right)$ for $\mathrm{Fe}_{8}$ with $B=0.046$ $\mathrm{K}$, and $D^{\prime} S_{z}^{4}$ for $\mathrm{Mn}_{12}$ with $D^{\prime}=-1.1 \times 10^{-3} \mathrm{~K}$. These lead to nondegeneracies for the lower levels that are comparable or bigger than the perturbations used to couple these levels. For $\mathrm{Mn}_{12}$, e.g., we obtain $E_{1}-E_{-2}=12 D^{\prime}$ when $h=h_{1}$, and $E_{1}-E_{-3}=48 D^{\prime}$ when $h=h_{2}$.

[9] A. L. Burin, N. V. Prokof'ev, and P. C. E. Stamp, Phys. Rev. Lett. 76, 3040 (1996); A. Fort et al., ibid 80, 612 (1998); D. A. Garanin and E. M. Chudnovsky, Phys. Rev. B 56, 11,102 (1997).

[10] F. Hartmann-Boutron, P. Politi, and J. Villain, Int. J. Mod. Phys. B 10, 2577 (1996).

[11] R. Sessoli, Mol. Cryst. Liq. Cryst. 274, 145 (1995).

[12] J. R. Friedman et al., J. Appl. Phys. 81, 3978 (1997).

[13] F. Lionti et al., J. Appl. Phys. 81, 4608 (1997).

[14] This argument would fail if the relaxation was already saturated at $0.5^{\circ}$ (A. J. Leggett, comment at seminar). This is unlikely to be so, because the jump at $H_{1}$ is very small, and less than $3 \%$ of the total $\Delta M$ for all $\theta \leq 10^{\circ}$.

[15] The names are based on the prefactors in $a_{j, j+1}$, and should not be interpreted too closely.

[16] It is quite likely that the "naive" rate $\Gamma^{0}(H)$ can not quantitatively describe $\mathrm{Mn}_{12}$ for any value of $H$ at low temperatures. The comparison made here serves only to prove the point that lattice distortion effects are large.

[17] At very low temperatures, the systematic increase in $\gamma_{1}^{4}$ from the decrease in $\epsilon_{21}$ may nullify this effect, but we do not have any hard calculations to support this point. 